\title{
Prevalence of Dental Crowding in the Kabul Dental Hospital, Kabul-Afghanistan
}

\author{
Ozair Erfan, Gulbahar Taka, and Hosna Qaderyar
}

\section{ABSTRACT}

Background: Dental crowding, also called overcrowding, is a condition in which there is not enough space in the mouth for the steady growth of permanent teeth. Common problems that crowding can cause for a person include difficulty chewing food, speech problems, tooth decay, enamel loss, premature tooth loss, pressure on the jaws and pain, gingival resorption, and tenderness. Crowding always creates an unpleasant appearance for a person and even causes a person to be isolated from society and reduce selfconfidence due to an ugly appearance. Crowding raises serious functional and aesthetic concerns for many patients.

Aims: Conduct a descriptive study in this field with 1000 patients of the (OPD) Outpatient Department of Kabul Dental Hospital on the prevalence of crowding and to finding the relationship between the incidence of crowding according to the upper and lower jaws and the anterior and posterior segments of each jaw.

Methods and materials: The sampling method were systematic random sampling that 1000 people were randomly selected from all OPD service clients during nine months and were included in the study. The required information was obtained by clinical examination of patients above the dental unit, under adequate light and the kit of dental examination equipment with direct clinical examination of the patient, and a preprepared questionnaire was inserted.

The information obtained from the questionnaire reached the coding page, and it was entered into IBM_SPSS-25 software, and its analysis was performed.

Results: From the results of this study, it was found that the prevalence of crowding was $59.1 \%$, with the highest incidence of $28.6 \%$ in the lower jaw. Also, the highest rate of crowding events was $44.9 \%$ in the anterior segment of the mandible. Crowding events were received in the upper jaw $12.7 \%$, with the highest incidence of $29.3 \%$ in the anterior segment.

Keywords: Crowding, Prevalence, Upper jaw, Lower jaw.
Published Online: June 30, 2021

ISSN: $2684-4443$

DOI: 10.24018 /ejdent.2021.2.3.65

Ozair Erfan*

Head of Oral and maxillofacial surgery department, Stomatology faculty, Herat University, Afghanistan.

(e-mail: ozairerfan ${ }^{\circledR}$ yahoo.com)

Gulbahar Taka

Teaching Assistant, Oral and maxillofacial surgery department, Stomatology faculty,

Herat University, Afghanistan.

Hosna Qaderyar

Teaching Assistant, Oral and maxillofacial surgery department, Stomatology faculty, Herat University, Afghanistan.

*Corresponding Author

\section{INTRODUCTION}

Crowding is one of the significant manifestations of class I malocclusion [1], [2]. Crowding is mainly due to the disproportion between the size of the teeth and the length of the dental arch [3], [4]. Crowding is defined by the amount of space required for the correct placement of the tooth and is achieved in two ways. 1) By measuring the amount of space required and reducing this amount by calculating the available space through the width of the teeth 2) By measuring the degree of overlap of the teeth (5). Crowding can be mild, moderate, or severe, depending on the size of the patient's jaw and the number of teeth (6).

Mild crowding of teeth is when an anterior tooth in the upper or lower jaw is slightly rotated.

Medium crowding of teeth is when two to three anterior teeth overlap in the upper or lower jaw and, Severe tooth crowding is when most of the anterior teeth of the upper or lower jaw overlap [6].

Various factors can be involved in creating crowding, including:

1. Disproportion between the length of the dental arch and the size of the teeth (reducing the length of the dental arch or increasing the size of the teeth). The mesiodistal diameter of group teeth containing crowding is significantly larger than the group without crowding, and also the width of the dental arch of the group containing crowding is significantly smaller than the group without crowding [5], [7], [8].

2 . The presence of extra teeth or supernumerary can lead to dental crowding [9].

3. Prolonged remains of deciduous teeth can cause permanent teeth to erupt in an abnormal position, leading to 
dental crowding [8].

4. The size and shape of the teeth can also lead to crowding [6].

5. Early loss of deciduous teeth due to deviation of teeth adjacent to the edentulous area can lead to crowding [5], [6].

For example, with the extraction of the second deciduous molars, space is provided for the mesial deviation of the permanent first molars, which belongs to the second premolars, which the molars will occupy.

6. Delayed growth of the mandible can be the cause of delayed crowding of the mandibular incisors. In this case, the delayed growth of the mandible causes the lower incisors to move distally and become crowding [10].

7. Research on the relationship between third molars and crowding shows that third molars do not make a statistically significant difference when comparing people with extracted, existing, or non-existent third molars [11], [12].

8. Crowding seems to be more than a tooth size problem. It has been shown that well-regulated dental arches in early adolescence can often show crowding in adulthood. This condition may also be seen after successful orthodontic treatment [3].

\section{Methods AND MATERIALS}

The research was conducted in the form of a descriptive study performed on 1000 clients of the OPD service of Kabul Dental Hospital.

The sampling method was systematic random sampling that 1000 people were randomly selected from all OPD service clients during nine months and were included in the study. The required information was obtained by clinical examination of patients above the dental unit, under adequate light and the kit of dental examination equipment with direct clinical examination of the patient, and a pre-prepared questionnaire was inserted.

The information obtained from the questionnaire reached the coding page, and it was entered into IBM_SPSS-25 software, and its analysis was performed. In this study, patients were included who had the following characteristics:

\section{A. Including Criteria}

1. They have not had any orthodontic treatment before.

2. The majority of teeth, especially the first molars and the anterior teeth of both jaws, are present.

3. Extensive restorative treatment and cosmetic or restorative treatment have not been performed on the crown of the anterior teeth and the first molar.

4 All patients are between the ages of 15-30.

\section{B. Excluding Criteria}

Patients older or younger than 15-30 years old were not included in the study. Only patients who have previously undergone orthodontic treatment, or have extensive crown repairs, typically in the anterior segment of the jaws, or many teeth, especially the first molars or six anterior teeth, have been extracted, or for whatever reason are not available, not included in the research.

\section{The Main Objective}

Receiving the prevalence of crowding events in the upper and lower jaws.

\section{Subjective}

1. Prevalence of malocclusion;

2. Receive the amount of crowding events according to the position of the jaws and anterior and posterior segments.

\section{RESULTS}

The following results were obtained in a study of 1000 clients of the OPD service of Kabul Dental Hospital, between the ages of 15-30 to obtain information about the prevalence of crowding and the relationship between its occurrence according to the upper and lower jaws and the anterior and posterior segments of each jaw.

In Fig. 1, the prevalence of dental crowding was described. It shows which the prevalence of dental crowding was $59.1 \%$ in all subjects.

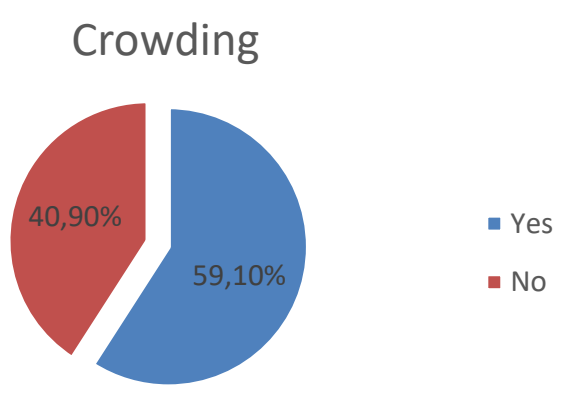

Fig. 1. Prevalence of dental crowding.

Regarding which jaw is more prone to crowding, it was found that the lower jaw is approximately 2.5 times more crowded than the upper jaw (Fig. 2).

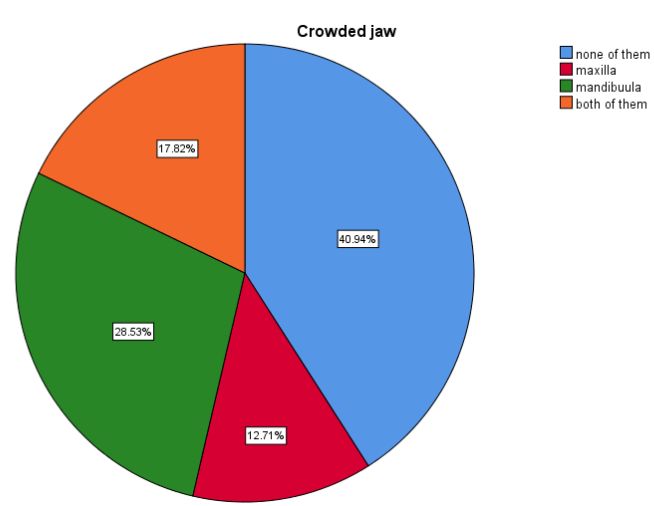

Fig. 2. Crowding according to the jaws.

In this graph, the rate of crowding events in the lower jaw is $28.6 \%$, approximately 2.5 times higher than the upper jaw $12.7 \%$. However, in $17.8 \%$ of cases, both jaws were exposed to crowding.

In the Table I described which jaw is more prone to crowding, it was found that the lower jaw with $28.6 \%$ is approximately 2.5 times more crowded than the upper jaw with $12.7 \%$. However, in $17.8 \%$ of cases, both jaws were exposed to crowding.

In Fig. 3. The following results were obtained as to which part of the upper jaw shows the most crowding. It is shown that crowding is mainly observed in the anterior segment of the maxilla $29.3 \%$. In other words, except for a small percentage of $1 \%$, all dental crowding events are observed in the anterior segment of the maxilla. 


\begin{tabular}{cccccc}
\multicolumn{6}{c}{ TABLE I: CROwDED JAW } \\
\hline & Frequency & Percent & Valid Percent & $\begin{array}{c}\text { Cumulative } \\
\text { Percent }\end{array}$ \\
\hline \multirow{4}{*}{ Valid } & none of them & 409 & 40.9 & 40.9 & 40.9 \\
& maxilla & 127 & 12.7 & 12.7 & 53.7 \\
& mandible & 285 & 28.5 & 28.5 & 82.2 \\
& both of them & 178 & 17.8 & 17.8 & 100.0 \\
Missing & Total & 999 & 99.9 & 100.0 & \\
& System & 1 & 0.1 & & \\
\hline & Total & 1000 & 100.0 & & \\
\hline
\end{tabular}

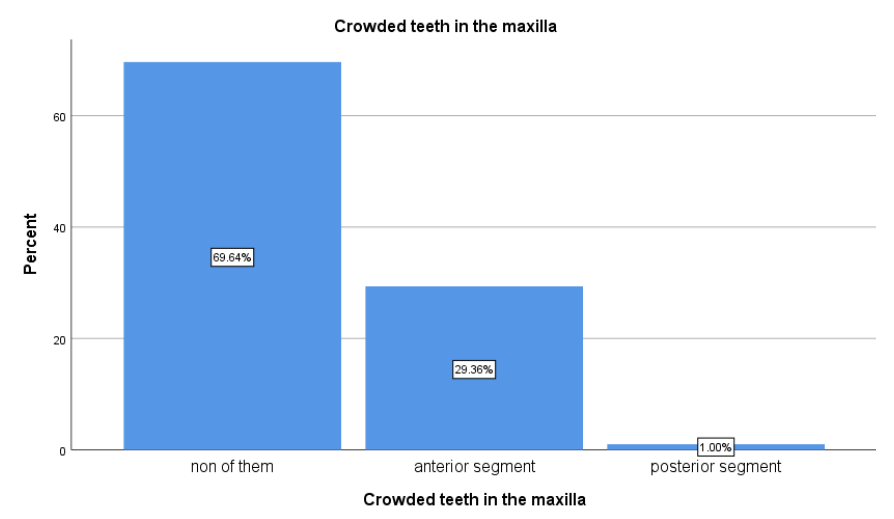

Fig. 3. Crowded teeth in the maxilla.

The following results were obtained as to which part of the lower jaw shows the most crowding (Fig. 4). In this figure, it can be seen that $44.9 \%$ of cases of dental crowding are observed in the anterior segment of the mandible, and a small percentage of $1.6 \%$ of dental crowding was received only in the posterior segment. $0.1 \%$ of dental crowding events were received in both segments, which is negligible.

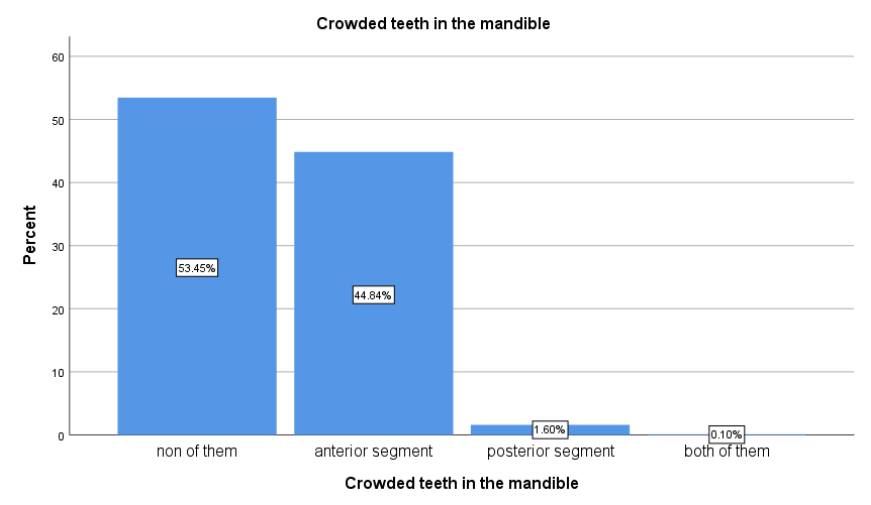

Fig. 4. Crowded teeth in the mandible.

\section{DISCUSSION}

The research concluded that the prevalence of dental crowding cases is $59.1 \%$, most of which is $49.9 \%$ in the anterior segment of the mandible. The findings of this study correspond with some studies conducted in other countries. In a study on the anterior segment of the mandible in the Tumkur population, which was performed on more than 120 people between the ages of 13-22, the anterior segment of the mandibula received $56.66 \%$. Of these, $43.33 \%$ had no dental crowding which described in Table II [10].

Another study in Saudi Arabia, in the city of Jeddah, found more than 1,064 women between the ages of 13 and 19. This table, which shows the results of research conducted in the city of Jeddah, Saudi Arabia, shows that the lower jaw is more crowded than the upper jaw which described in Table III [13]

TABLE II: PREVALENCE OF CROWDING IN TUMKUR POPULATION [10]

\begin{tabular}{cc}
\hline Prevalence & Jaw \\
\hline $6,8 \%$ & Maxilla \\
$31,9 \%$ & Mandibula \\
$56,66 \%$ & Both of them \\
$43,3 \%$ & Non \\
\hline
\end{tabular}

TABLE III: PREVALENCE OF CROWDING IN SAUDI ARABIA [13]

\begin{tabular}{cc}
\hline Prevalence & Jaw \\
\hline $7.6 \%$ & Maxilla \\
$13.2 \%$ & Mandibula \\
$60.6 \%$ & Both of them \\
$18.6 \%$ & Non \\
\hline
\end{tabular}

In a study of Pattern of dental crowding over 168 people in Tokyo, Japan, they found that the anterior segment of the mandible has the highest rate of crowding [6].

\section{CONCLUSION}

From 1000 OPD patients in National and Specialized Stomatology Hospital, 59.1\% had dental crowding, while $40.9 \%$ were normal means without dental crowding. Which the incidence was more in the lower jaw 28.6\%. Also, the highest rate of crowding events was in the anterior segment of the mandible $44.9 \%$.

\section{REFERENCES}

[1] Antanas S, Giedre T. Effect of the lower third molars on the lower dental arch crowding. Stomatologija. 2006;8(3):80-4.

[2] Erfan O, Khan M, Sina MN. Prevalence of dental malocclusion in stomatology hospital, Kabul-Afghanistan. IP Indian Journal of Orthodontics and Dentofacial Research. 2020;6(2):82-5.

[3] Brown VP, Daugaard-Jensen I. Changes in the dentition from the early teens to the early twenties: a longitudinal cast study. Acta Odontologica Scandinavica. 1951;9(3-4):177-92.

[4] Erfan O, Rahmani MH, Taka G. Prevalence of midline diastema according to race in Afghanistan. IP Indian Journal of Orthodontics and Dentofacial Research. 2020;6(4):241-4.

[5] Proffit WR, Fields HW, Msd DM, Larson B, Sarver DM. Contemporary Orthodontics, 6e: South Asia Edition-E-Book: Elsevier India; 2019.

[6] Shigenobu N, Hisano M, Shima S, Matsubara N, Soma K. Patterns of dental crowding in the lower arch and contributing factors: a statistical study. The Angle Orthodontist. 2007;77(2):303-10.

[7] RIEDEL RA. A postretention evaluation. The Angle Orthodontist. 1974;44(3):194-212.

[8] Richardson ME. The etiology of late lower arch crowding alternative to mesially directed forces: a review. American Journal of Orthodontics and Dentofacial Orthopedics. 1994;105(6):592-7.

[9] Jawale B. Effect of Asymmetric Premolar Extractions on Smile Aesthetics in A Patient With Severe Crowding. A Case Report, SAR J Dent Oral Surg Med. 2021;2(3):36-43.

[10] Madhusudhan V, Mahobia Y. Prevelance of mandibular anterior crowding in tumkur population. J Dent Sci Res. 2011;2(2):6-8.

[11] Jacobson L, Linder-Aronson S. Crowding and gingivitis: a comparison between mouthbreathers and nosebreathers. European Journal of Oral Sciences. 1972;80(6):500-4.

[12] Chang H, Shiau Y, Chen K. The relationship of dental crowding to tooth size, dental arch width, and arch depth. Proceedings of the National Science Council, Republic of China Part B, Life sciences. 1986;10(4):229-35.

[13] Al-Hummayani FM. Prevalence of incisors crowding in Saudi Arabian female students. Cairo Dent J. 2004;20:413-6. 